\title{
Molecular dynamics of coalescence and collisions of silver nanoparticles
}

\author{
Enrique Guevara-Chapa $\cdot$ Sergio Mejía-Rosales
}

Received: 3 March 2014 / Accepted: 13 November 2014/Published online: 4 December 2014

(C) Springer Science+Business Media Dordrecht 2014

\begin{abstract}
We study how different relative orientations and impact velocity on the collision of two silver nanoparticles affect the first stages of the formation of a new, larger nanoparticle. In order to do this, we implemented a set of molecular dynamics simulations on the NVE ensemble on pairs of silver icosahedral nanoparticles at several relative orientations, that allowed us to follow the dynamics of the first nanoseconds of the coalescence processes. Using bond angle analysis, we found that the initial relative orientation of the twin planes has a critical role on the final stability of the resulting particle, and on the details of the dynamics itself. When the original particles have their closest twins aligned to each other, the formed nanoparticle will likely stabilize its structure onto a particle with a defined center and a low surface-to-volume ratio, while nanoparticles with misaligned twins will promote the formation of highly defective particles with a high inner energy.
\end{abstract}

E. Guevara-Chapa ( $\square)$

Facultad de Ciencias Físico Matemáticas, Universidad Autónoma de Nuevo León,

66450 San Nicolás de los Garza, NL, Mexico

e-mail: enrique_guevara@hotmail.com

S. Mejía-Rosales

Center for Innovation, Research and Development in Engineering and Technology (CIIDIT), and CICFIMFacultad de Ciencias Físico Matemáticas, Universidad Autónoma de Nuevo León,

66450 San Nicolás de los Garza, NL, Mexico
Keywords Molecular dynamics · Nanoparticles · Silver $\cdot$ Coalescence $\cdot$ Embedded atom $\cdot$ Modeling and simulations

\section{Introduction}

In the last decade, the possibility of fine-tuning the physical, chemical, and optical properties of metal nanoparticles by the manipulation of their sizes and geometries has enormously increased the interest of scientists and engineers in these systems. Depending on the technique used to produce the particles and on the specific material being used, metal nanoparticles may be tailor-made for applications as varied as biofiltering, colloidal chemistry, catalysis, electrochemistry, heat dissipation, and medicine. Kim et al. (2007), Morones et al. (2005), Haes and Duyne (2002), McFarland and Duyne (2003) and Silva et al. (2011)

Unlike the first chemical-based techniques developed for the production of metal nanoparticles, the use of inert gas condensation reactors allows to create particles by the direct interaction of small clusters, without the need of additional reactants, and in the absence of a substrate that affects the initial stages of the synthesis. Thus, the main features of the resulting particles are mostly due to the kinematics that take place on the synthesis reactor, on condition that the eventual deposition on a substrate is made within the soft-landing regime Gracia-Pinilla et al. (2009), and that there are 
not large external excitations once the particle is on the substrate (as, for example, an intense electron beam from a transmission electron microscope).

Among the different metals commonly used in the production of nanoparticles, silver has a particularly important place, both because its relative abundance, and because its wide range of employment, that goes from bactericides Sharma et al. (2009), Morones et al. (2005) to plasmonics Cobley et al. (2009) and thin-film photovoltaics Silva et al. (2011). A common trend to all these different uses is the criticality of sizes and shapes of the nanoparticles. It is known, for example, that the wavelength of the localized surface plasmon resonance (LSPR) depends on the shape of the particle Cobley et al. (2009), and that the inhibitory effects of silver nanoparticles on the growth of bacteria vary with the size of the particles Rai et al. (2009).

The generation of metal nanoparticles in many of the synthesis methods, but particularly in the synthesis using an inert gas condensation reactor, involves the collision between smaller particles formed in earlier stages of the process. In this method, a supersaturated vapor of individual atoms, dimers, and small clusters is generated by the sputtering of material from a metal target, and the sputtered material nucleates into small particles aided by the pressure of an inert gas on the synthesis chamber. Depending on the length of the chamber and the applied accelerating voltage, the small particles will collide with their neighbors, generating larger particles Mariscal et al. (2005). This collision mechanism has been studied by molecular dynamics (MD) simulations in silicon Hawa and Zachariah (2005), Hawa and Zachariah (2006), gold Antúnez-García et al. (2011), and between particles of different metals Mariscal et al. (2005). These studies have confirmed that the specifics of the kinetic initial conditions play a defining role in the kind of structure that the final nanoparticle takes.

In this paper, we study how different relative velocities and orientations on the collision of two silver nanoparticles affect the first stages of the formation of a new, larger nanoparticle. In order to do this, we implemented a set of MD simulations on the NVE ensemble, which allowed us to follow the dynamics of the first nanoseconds of the coalescence process. This work is aimed at producing a better understanding of the kinematic processes that take place when two particles find each other at the inert gas condensation chamber or at the surface of a substrate where the substrate-metal interactions are weak. The paper is organized as follows: "Models and calculations" section describes the MD parameters and interaction model used in our calculations, and the initial structures used in the simulations. In "Results" section, we present the results for the different structures and models. Finally, "Conclusions" section discusses the results of our calculations and summarizes the main conclusions.

\section{Models and calculations}

\section{The MD model}

MD is known to be a tool of great reliability in the study of metallic structures Baletto et al. (2001), when it is used with an appropriate interaction potential, since a suitable choice of interactions in the simulations will reproduce quantitatively the main properties measured by experiments, and, depending on the detail of the models and the choice of thermodynamic conditions, will qualitatively match the behavior of other properties. In this work, we simulated the particle-particle collisions by the use of micro canonical MD, with a Verlet algorithm. Each production run was 10 ns long, with a time step of $1 f s$. That time is enough to stabilize the atomic configuration after a $500 \mathrm{~m} / \mathrm{s}$ coalitions as shown in Fig. 4 .

The original particles were independently thermalized at $300 \mathrm{~K}$ previous to the collision, and the drift velocity to make the particles collide against each other was given by adding it to each atomic velocity that resulted from the thermalization process. The atom-atom interactions were calculated by the use of the embedded-atom interaction model, as implemented by Foiles et al. Foiles et al. (1986). Here, the total energy of the system is given by considering the contribution of all the atoms:

$$
E=\sum_{i} E_{i},
$$

where

$$
E_{i}=\frac{1}{2} \sum_{i j} \phi_{i j}\left(r_{i j}\right)+\sum_{i} F_{i}\left(\rho_{i}\right) .
$$

Here, the first term corresponds to the pairwise interaction between atoms, while the second term accounts for the energy needed to embed an atom in 
the $i$-th site that has an electron density $\rho_{i}$. As it is well known, the modeling of metals requires the inclusion of this kind of non-pairwise terms in order to reproduce adequately the elastic properties of the system.

The collision model

The original particles were icosahedral, each particle made of 2,057 atoms, which corresponds to eight full icosahedral shells surrounding a central atom. The icosahedral shape was selected due to its common appearance on silver (and other metal) particles at this range of sizes (Perez-Tijerina et al. 2010), and because with this choice, a small number of nonequivalent orientations can be used to form the set of starting configurations. Ten different orientations were considered in this study: (i) edge-to-edge, (ii) edge-to-face, (iii) edge-to-vertex, (iv) edge-to-random, (v)face-to-face, (vi) face-to-vertex, (vii) face-torandom, (viii) vertex-to-vertex, (ix) vertex-to-random, and (x) random-to-random. These configurations are shown in Fig.1.

The structures were generated using the nominal interatomic distance of $\mathrm{Ag}$ in bulk, and were thermalized individually running MD simulations on the canonical ensemble, at $300 \mathrm{~K}$, followed by a short NVE simulation to confirm the thermalization. Once thermalized, each pair of particles was created at a distance larger that the range of the interatomic potential. Each set of initial conditions was used to generate three MD production runs at the same conditions, where the only difference between them was the random distribution of initial velocities.

Different velocities were tested for each orientation, ranging from $0 \mathrm{~m} / \mathrm{s}$ (pure coalescence at contact) to $500 \mathrm{~m} / \mathrm{s}$; this range of velocities covers the typical conditions at which the particles collide in an inert gas condensation process (Perez-Tijerina et al. 2010). It was found that in this range only the velocity of 500 $\mathrm{m} / \mathrm{s}$ gave the particles the enough kinetic energy to deform the original nanoparticles to promote the creation of a new nanoparticle, with a well-defined center. For this reason, this study is centered in the results obtained at this velocity of impact.

Analysis methods

For purposes of data analysis, the temperature and potential energy of the systems were monitored along the simulations. The individual atomic positions, velocities and net forces were monitored as well. The atomistic trajectories of each run were used to investigate the atomic packing using an Ackland analysis, since this method, based on the statistics of the angles formed by the vectors defined by the positions of neighboring atoms, is more stable to thermal fluctuations than other methods, such as the common neighbor analysis method Ackland and Jones (2006).

The final configuration of each production run was used as input for the simulation of high angle annular dark field scanning transmission electron microscopy
Fig. 1 Starting

configurations used in the MD simulations. a Edgeedge, $\mathbf{b}$ edge-face, $\mathbf{c}$ edgevertex , d edge-random, e face-face, $\mathbf{f}$ face-vertex, g face-random, $\mathbf{h}$ vertexvertex, i vertex-random, j random-random. Colors are used just to identify the primitive particles. (Color figure online)

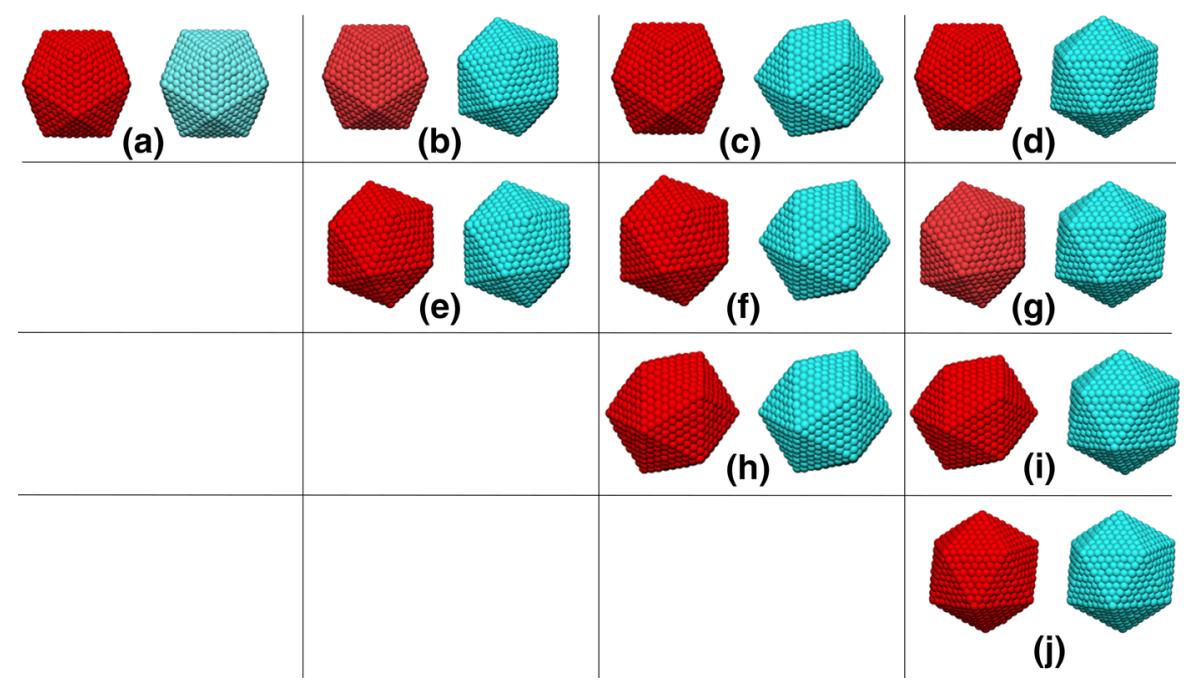


(HAADF-STEM) images. These images were calculated taking into consideration that in HAADF-STEM real micrographs of thin specimens (this is, thickness on the order of the size of the particles considered in this study), the intensity due to an atomic column formed by $n$ atoms is approximately given by

$$
I \propto \sum_{i=1}^{n} Z_{i}^{k}
$$

where $Z_{i}$ is the atomic number of the $i$-th atom, and $k$ is a number that we set to be equal to 1.37 , based on previous results obtained using a proper calculation of the interaction of the specimen with the electron beam of the microscope Khanal et al. (2013). In this way, the simulated micrographs can be justly compared against real high resolution HAADF-STEM micrographs.

\section{Results}

In order to analyze the influence of initial relative velocities on the dynamics of the coalescence process, several impact velocities were tried. It was found that, for the size of the particles considered in this study, at impact velocities of zero (pure coalescence at contact), $100 \mathrm{~m} / \mathrm{s}$, and even $300 \mathrm{~m} / \mathrm{s}$, the energy available from the impact to rearrange the atomic structure of the particles is not enough to form a new, recognizable particle with a well-defined center. This can be compared against previous studies on impacts between smaller particles, such as the one made by Mariscal et al. , where two metal particles made of 95 atoms each can be made to coalesce using an impact velocity of $100 \mathrm{~m} / \mathrm{s}$ Mariscal et al. (2005). Unlike the case of this study, in the systems treated by Mariscal et al. the surface-to-volume ratio on the particles is high enough to promote coalescence in order to lower the surface energy. In the particles considered in this study, one of the first evident facts detected when comparing the results at zero velocity with the results at 100 and 300 $\mathrm{m} / \mathrm{s}$ was that at this range velocity there is no significative difference between the dynamics of systems otherwise prepared identically. Figure 2 shows clearly this trend for the particular case of the edge-edge relative orientation. The figure compares the structure of the systems at 0,100 , and $300 \mathrm{~m} / \mathrm{s}$, at five different times of the coalescence process. The cross section of the particles are shown, with the colors of the atoms representing the local ordering as calculated by Ackland analysis: red atoms correspond to twin planes, green atoms represent FCC structure, white atoms do not correspond to any particular arrangement, and blue atoms are supposed to be related to BCC packing, but since these atoms are not grouped, this interpretation is not correct and hence blue atoms, as the white ones, should be interpreted just as disordered structure. It can be clearly seen that in the three cases, the icosahedral motif of the original particles is basically conserved, with the disordered volume concentrated around the impact region. After a few nanoseconds of dynamics, the disordered regions had shrinked to a small volume, and the centers of the original particles are still easily localized in the structure. A general trend is observed by monitoring the evolution of the configurational energy of the system (see Fig. 3). Immediately after the two original particles met, there is a slight drop on the average configurational energy, due to the rearrangement of the surface atoms close to the contact area, and to the increase on the average coordination number, which implies a rise on the number of pair interactions. After this transitory stage, the conversion of translational into thermal into potential energy dominates the process, and the potential energy increases in an almost linear fashion due to the loss of structure in the particle at the vicinity of the contact area. After this linear increase, the potential energy drops slightly as the surface atoms reach their nearest potential wells, and the particle increases its temperature about $30 \mathrm{~K}$. Figure 3 shows that the transitory stage takes around 50,000 MD steps, or 5 ps.

In Fig. 4, the potential energy of whole system is shown. It is clear that after the fist nanosecond the system has reach equilibrium and that the simulation is long enough to consider the final state as a permanent state of the system.

A second observation was that, despite the fact that the motion of an atom originally at the surface to the inner part of the particle that results from the coalescence does not involve a change in energy (since all the atoms are from the same chemical species), there is a marked tendency of the surface atoms to remain in the surface even after coalescence. Figure 5 depicts several stages of the system after an impact at $300 \mathrm{~m} / \mathrm{s}$; here, the atoms have been colored according to the layer in which they are placed in the original icosahedra. As can be noted, even at this 
Fig. 2 Snapshots at different evolution times of the coalescence process at three different impact velocities. Upper row $0 \mathrm{~m} / \mathrm{s}$, middle row $100 \mathrm{~m} / \mathrm{s}$, lower row $300 \mathrm{~m} / \mathrm{s}$. Red atoms correspond to twin planes, green atoms represent FCC structure, and white and blue atoms are interpreted as disordered structure. (Color figure online)

Fig. 3 Typical time evolution of the configurational energy for a system of two particles impacting each other at 100 $\mathrm{m} / \mathrm{s}$

Fig. 4 Typical time evolution of the configurational energy for a system of two particles impacting each other at 500 $\mathrm{m} / \mathrm{s}$. The time covers the whole range of the simulation; impact occurs on the first $10 \mathrm{ps}$ of the dynamics (a)
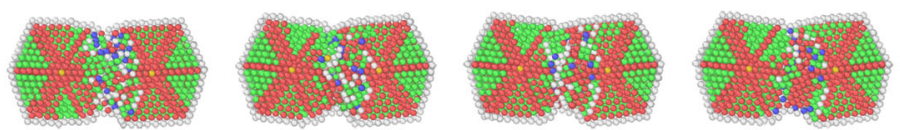

0.1 (ns)

$\mathrm{v}=0(\mathrm{~m} / \mathrm{s})$

$1.0(\mathrm{~ns})$
$\mathrm{v}=0(\mathrm{~m} / \mathrm{s})$

$2.0(\mathrm{~ns})$

$5.0(\mathrm{~ns})$

$\mathrm{v}=0(\mathrm{~m} / \mathrm{s})$

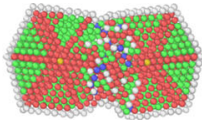

10.0 (ns) $\mathrm{v}=0(\mathrm{~m} / \mathrm{s})$

(b)
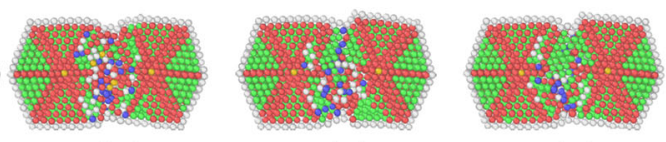

0.1 (ns)

$\mathrm{v}=100(\mathrm{~m} / \mathrm{s})$

$1.0(\mathrm{~ns})$

$\mathrm{v}=100(\mathrm{~m} / \mathrm{s})$

$2.0(\mathrm{~ns})$

$\mathrm{v}=100(\mathrm{~m} / \mathrm{s})$

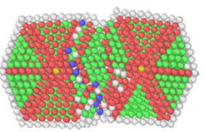

$5.0(\mathrm{~ns})$
$\mathrm{v}=100(\mathrm{~m} / \mathrm{s})$

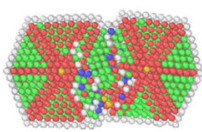

10.0 (ns)

$\mathrm{v}=100(\mathrm{~m} / \mathrm{s})$

(c)
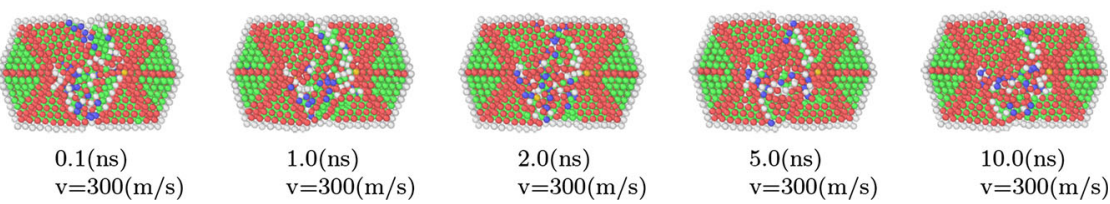
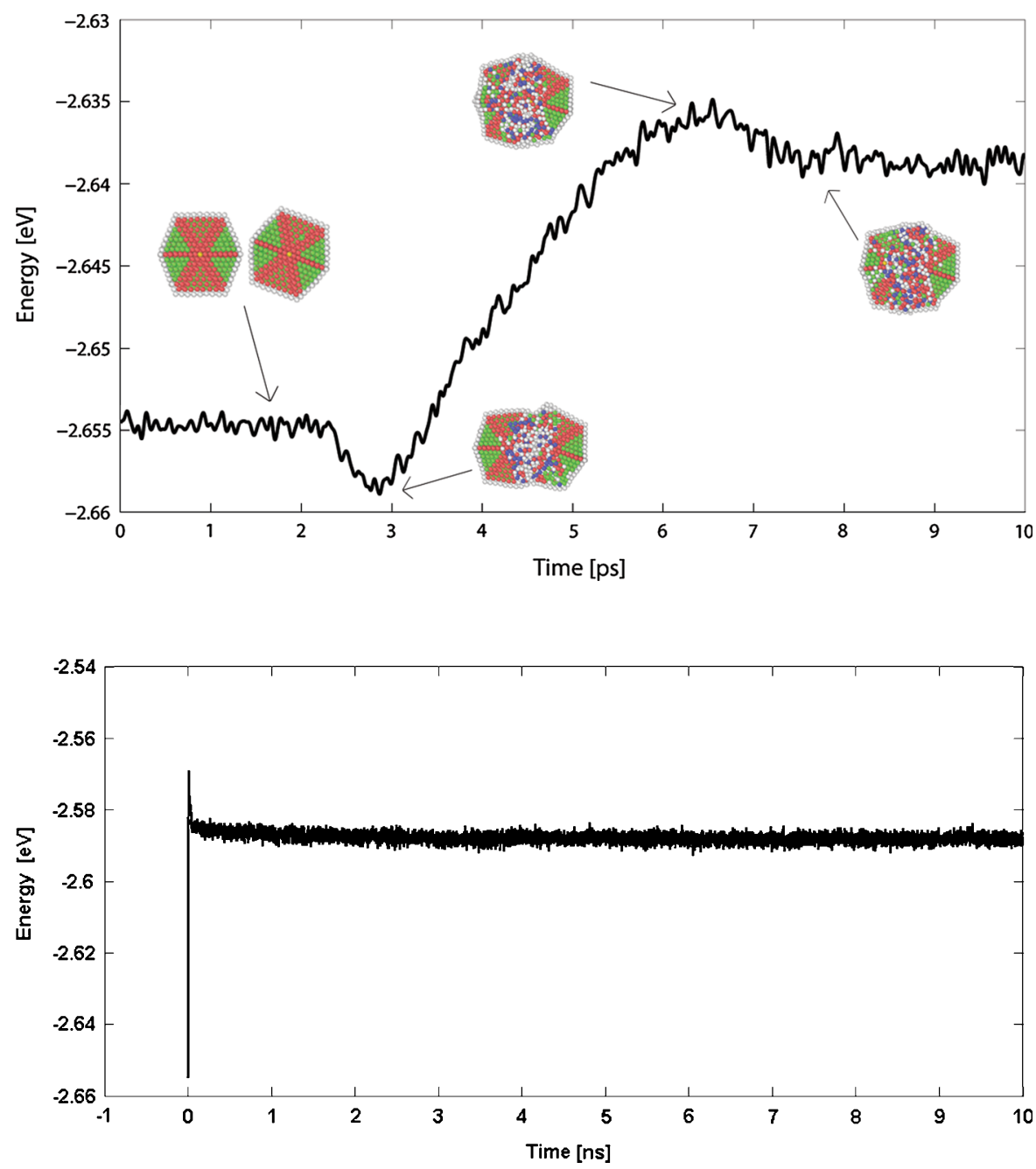


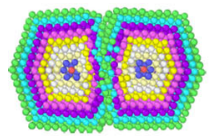

0.1 (ns)

$\mathrm{v}=300(\mathrm{~m} / \mathrm{s})$

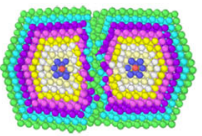

1.0 (ns)

$\mathrm{v}=300(\mathrm{~m} / \mathrm{s})$

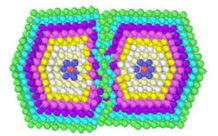

$2.0(\mathrm{~ns})$

$\mathrm{v}=300(\mathrm{~m} / \mathrm{s})$

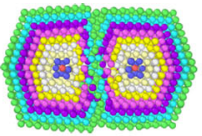

$5.0(\mathrm{~ns})$

$\mathrm{v}=300(\mathrm{~m} / \mathrm{s})$

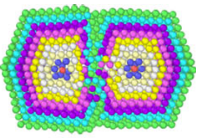

$10.0(\mathrm{~ns})$

$\mathrm{v}=300(\mathrm{~m} / \mathrm{s})$

Fig. 5 Snapshots at different evolution times of two coalescencing particles at relative velocity of $300 \mathrm{~m} / \mathrm{s}$. The colors identify the layers in which the atoms are placed in the original icosahedra. (Color figure online)

impact velocity the atom diffusion is almost nil. It was also found that at this range of velocities the particles tend to keep their relative orientations, except for the case of vertex-edge and face-edge impacts, where there is a remarked tendency of the particles to rotate in order to increase their contact area.

The phenomenology becomes more diverse when the relative impact velocity is $500 \mathrm{~m} / \mathrm{s}$. This is understandable considering that at this impact velocity, the kinetic energy per atom is several times grater that $0.1 \mathrm{eV}$, and thus it can be considered out of the softlanding regime. Cross sections of representative stages of the systems at different simulation times are represented in Fig. 6; here, the colors are again used to identify the layer at which the atoms were positioned in the original icosahedral particles. As can be seen, while the diffusion of the atoms is considerably higher than in the previous cases, the diffusion is nevertheless confined to the region directly adjacent to the area of impact, even in the systems that get deformed to such a degree that the final structure is a new particle with a well-defined center. As in the cases of a smaller impact velocity, at $500 \mathrm{~m} / \mathrm{s}$, the regions farther to the contact region keep their original structure, but, depending on the relative orientation of the original icosahedra, two twins may interact to form a new borderline between fcc regions, or may embed one into the other to make a larger twin. At all cases, at this impact velocity, after less than one nanosecond of dynamics there is no discernible borderline separating the two original particles, unlike what occurs to the particles at 300 $\mathrm{m} / \mathrm{s}$ (see Fig. 5). The kinetic energy distribution is not uniformly distributed along the particle, but it is mostly concentrated on a confined region of the particle that takes a liquidlike ordering and that commonly includes atoms of the surface. An overall tendency is that the greater the difference between the areas which come into contact when the primitive particles meet, the greater the diffusion on the resulting particle. Thus, the greater diffusion takes place when a vertex of one of the particles impacts a face of the other particle.

Another consequence of the fact that the particles do not reach a liquidlike state is that, irrespective of the initial configuration, the surface of the new particle is formed by almost the entirety of the atoms originally at the surface of the primitive particles. The process is sketched in Fig. 7, where the black regions represent the volume occupied by atoms originally at the surface. Approximately $0.1 \mathrm{~ns}$ after the impact, a neck is formed between the original particles, but the most external shell has opened, and the atoms closer to the impact region start to migrate to the surface forced by the pressure exerted by the colliding cores; after less than one nanosecond, the displaced atoms have formed a second layer at the surface of the particle, with just some atoms originally at the surface remaining close to the impact region.

We found that at $500 \mathrm{~m} / \mathrm{s}$, each original configuration generates a qualitatively different final nanoparticle in terms of structural stability, configurational energy, ratio surface/volumen, and presence of [111] facets. In order to make a classification of the particles according to their structural features, we record the configurational energy of each case, also we considered whether a final configuration had a well-defined center where all the twin planes converge, or not. We also considered the ratio of number of atoms on the surface against the number of internal atoms. This information is shown in Table 1 . The table shows that structural stability and the configurational energy is directly related with the amount of atoms on fcc packing, the orientation of the twin planes, and the [111] facets on the final nanoparticle. As it was expected, the nanoparticles with small ratio of surface/ internal atoms have a well-defined center and will have a lower configurational energy. The Ackland analysis shows that the relative orientation of the twin planes forming the primitive particles play a defining 
Fig. 6 Snapshots at different evolution times of a two coalescencing particles at relative velocity of $500 \mathrm{~m} / \mathrm{s}$. Red atoms correspond to twin planes, green atoms represent FCC structure, and white and blue atoms are interpreted as disordered structure. (Color figure online) (a)
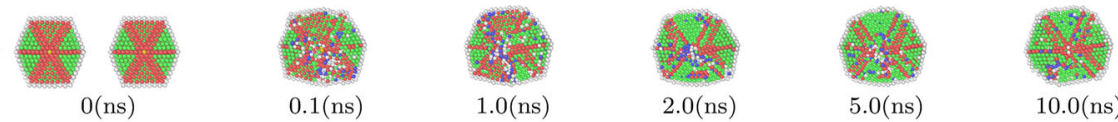

(b)

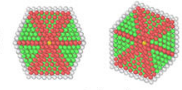

O(ns)
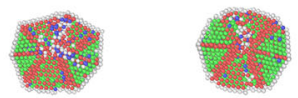

0.1 (ns)

$1.0(\mathrm{~ns})$

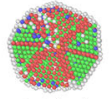

$2.0(\mathrm{~ns})$

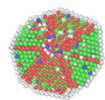

5.0 (ns)

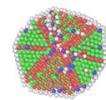

$10.0(\mathrm{~ns})$

(c)
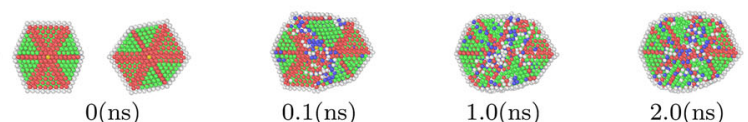

2.0 (ns)

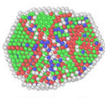

$5.0(\mathrm{~ns})$

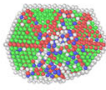

$10.0(\mathrm{~ns})$

(d)
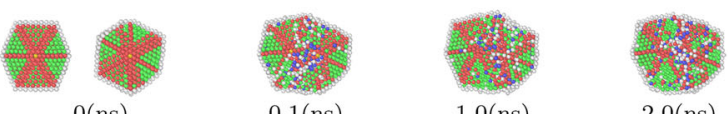

$1.0(\mathrm{~ns})$

$2.0(\mathrm{~ns})$
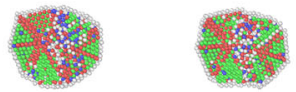

5.0 (ns)

$10.0(\mathrm{~ns})$

(e)
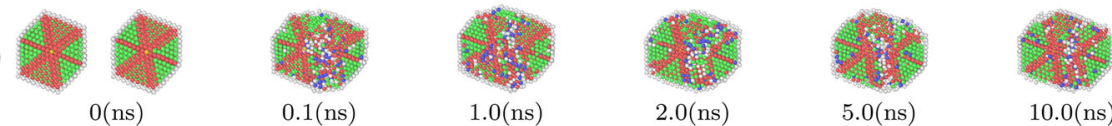

0.1 (ns)

$1.0(\mathrm{~ns})$

$2.0(\mathrm{~ns})$

$5.0(\mathrm{~ns})$

10.0(ns)

(f)
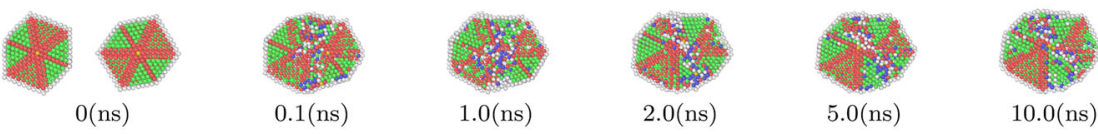

(g)
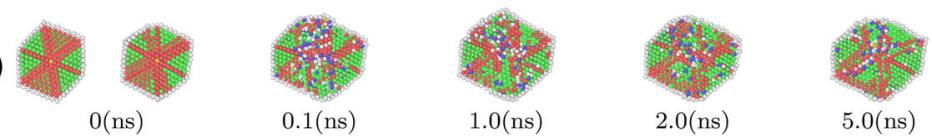

5.0 (ns)

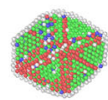

10.0(ns)

(h)
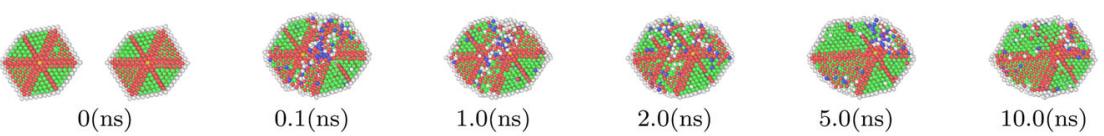

$2.0(\mathrm{~ns})$

$5.0(\mathrm{~ns})$

$10.0(\mathrm{~ns})$

(i)
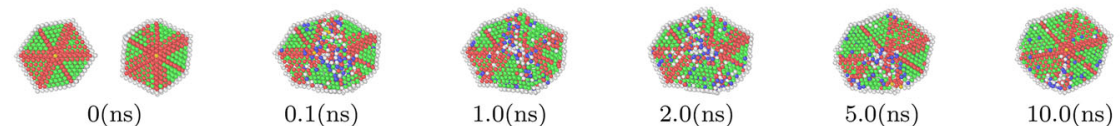

(j)
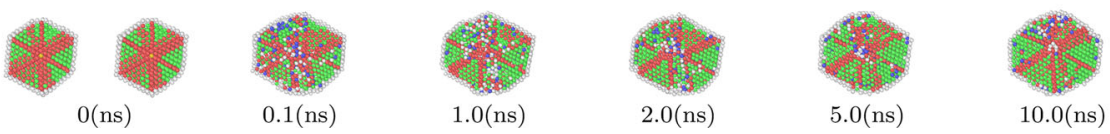

10.0(ns)
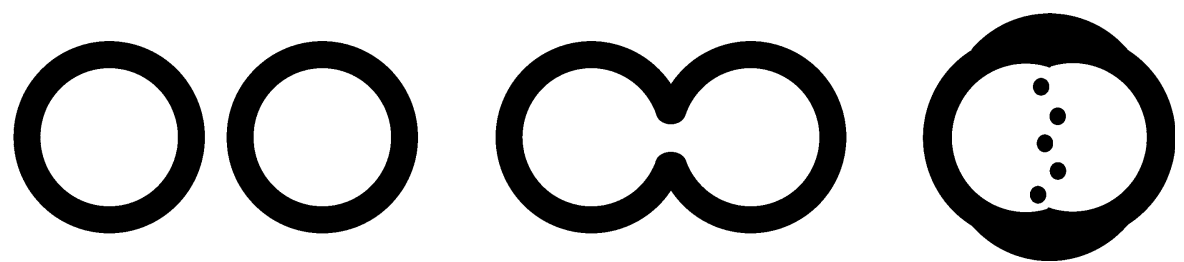

Fig. 7 Evolution of the surface of the particles colliding at $500 \mathrm{~m} / \mathrm{s}$. The black regions represent the most external shell of the primitive particles, at three different moments of the dynamics: before the collision (left), after $0.1 \mathrm{~ns}$ (center), and after $1 \mathrm{~ns}$ (right) of the impact 
Table 1 The table shows if the new nanoparticle has a well-defined center or not, the value of the surface to internal atoms ratio and the energy per atom for the final configuration of each relative orientation

\begin{tabular}{|c|c|c|c|c|}
\hline & Edge & Face & Vertex & Random \\
\hline Edge & $\begin{array}{l}\text { Well-defined center, } 45 \text { surf/ } \\
\text { iner }-2.5907[\mathrm{eV} / \text { atom }]\end{array}$ & $\begin{array}{l}\text { Well-defined center, } 45 \text { surf/ } \\
\text { iner }-2.5890[\mathrm{eV} / \text { atom }]\end{array}$ & $\begin{array}{l}\text { Not well defined, } 51 \text { surf/ } \\
\text { iner }-2.5881[\mathrm{eV} / \text { atom }]\end{array}$ & $\begin{array}{l}\text { Not well defined, } 50 \text { surf/ } \\
\text { iner }-2.5871[\mathrm{eV} / \text { atom }]\end{array}$ \\
\hline Face & & $\begin{array}{l}\text { Not well defined, } 46 \text { surf/ } \\
\text { iner }-2.5883[\mathrm{eV} / \text { atom }]\end{array}$ & $\begin{array}{l}\text { Not well defined, } 48 \text { surf/ } \\
\text { iner }-2.5873[\mathrm{eV} / \text { atom }]\end{array}$ & $\begin{array}{l}\text { Well-defined center, } 44 \text { surf/ } \\
\text { iner }-2.5898[\mathrm{eV} / \text { atom }]\end{array}$ \\
\hline Vertex & & & $\begin{array}{l}\text { Well-defined center, } 41 \text { surf/ } \\
\text { iner }-2.5896[\mathrm{eV} / \text { atom }]\end{array}$ & $\begin{array}{l}\text { Well-defined center, } 43 \text { surf/ } \\
\text { iner }-2.5911[\mathrm{eV} / \text { atom }]\end{array}$ \\
\hline Random & & & & $\begin{array}{l}\text { Well-defined center, } 42 \text { surf/ } \\
\text { iner }-2.5909[\mathrm{eV} / \text { atom }]\end{array}$ \\
\hline
\end{tabular}

Fig. 8 In a the horizontal twin planes of both nanoparticles are coplanar before the coalescence, and in $\mathbf{b}$ the new nanoparticle has a well-defined center where the twin planes converge. In $\mathbf{c}$ none of the twin planes on the original nanoparticles are coplanar, and as a consequence, and in d the new nanoparticle does not have a well-defined center and the twin planes do not converge into a common origin

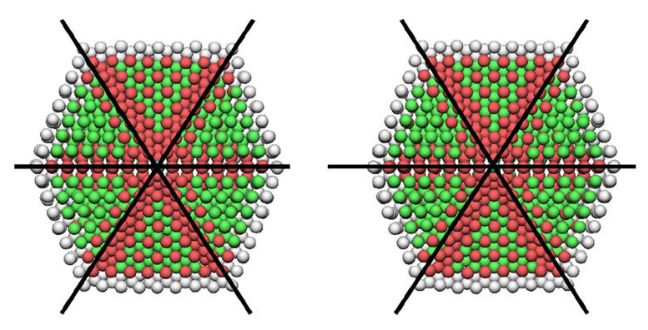

(a)

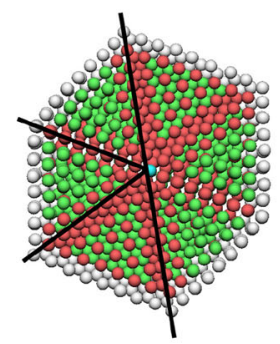

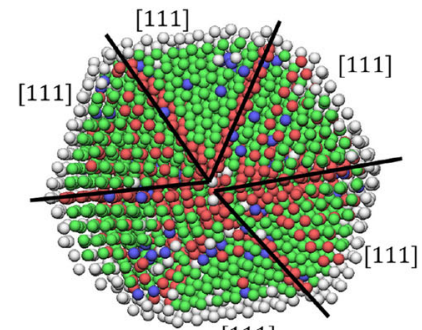

(b)

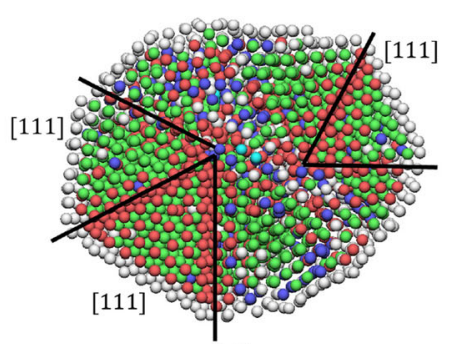

(d) role in the final structure of the particle. When a twin plane of one of the particles is almost aligned with a twin plane of the other particle, the particles rotate slightly in order to let the twins to align to each other and to form one plane. The relative orientation of the original twins also determines whether the resulting particle has a well-defined center or not, or if two adjacent tetrahedra can merge as a unique fcc region in the particle. When the twin planes are oriented such that the opposite ends extend in the same planes as shown in Fig. 8a, the new nanoparticle will have a well-defined center and mostly facets [111] as shown in Fig. 8b. If the twin planes are not aligned, as shown in Fig. 8c the new nanoparticle will not have a well- defined center and it will have an amorphous structure and only half of the surface as a [111] lattice as shown in Fig. 8. The explanation of the specific mechanism of interaction between twin planes while two particles collide is still at work in progress that will require additional sets of simulations and theoretical analysis.

High resolution aberration-corrected HAADFSTEM imaging is currently widely used to investigate the structure of metal nanoparticles, mainly because of its resolution power and its marked intensity dependence on the atomic number of the elements present in the particles. Having this in mind, we used our MD simulations results as input for the simulation of STEM micrographs, and the images are shown in Fig. 9. The 


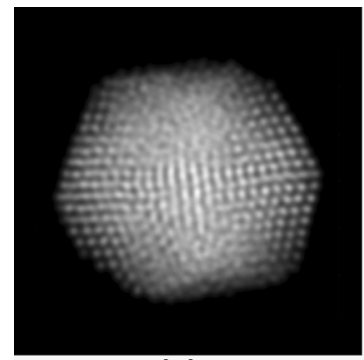

(a)

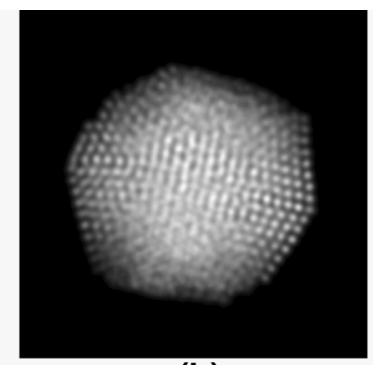

(b)

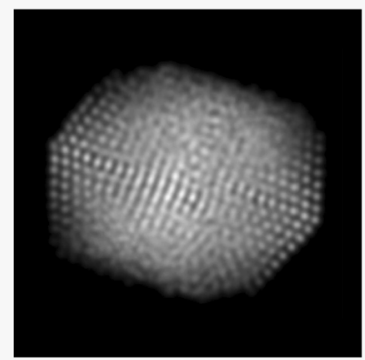

(e)

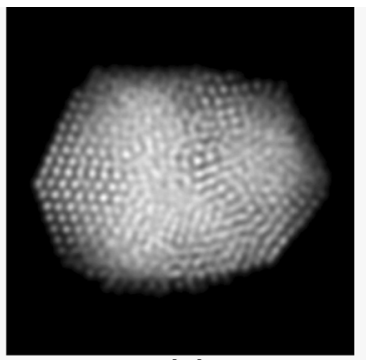

(c)

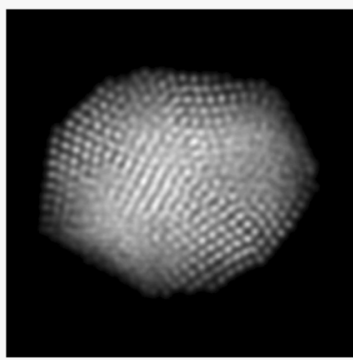

(f)

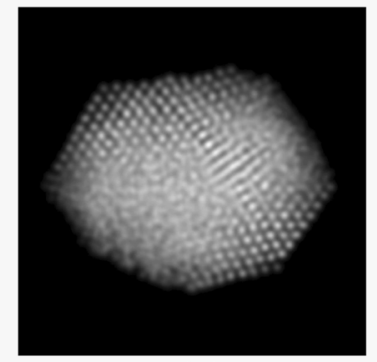

(h)

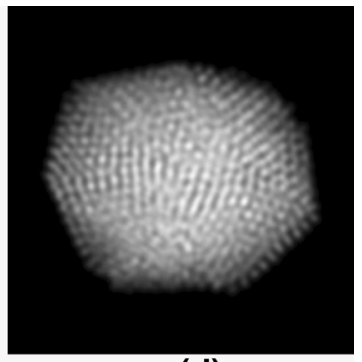

(d)

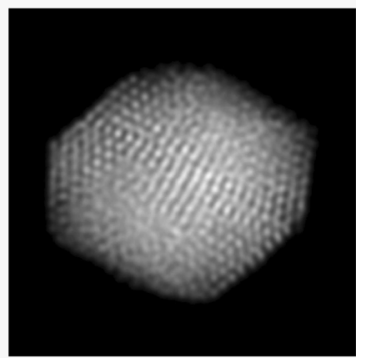

(g)

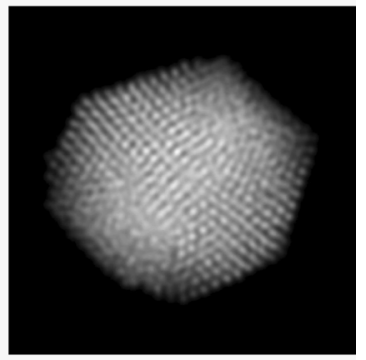

(i)

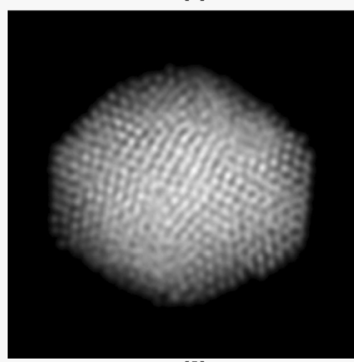

(j)

Fig. 9 Final configurations visualized as STEM images. a Edge-edge, $\mathbf{b}$ edge-face, $\mathbf{c}$ edge-vertex, $\mathbf{d}$ edge-random, $\mathbf{e}$ face-face, $\mathbf{f}$ facevertex, $\mathbf{g}$ face-random, $\mathbf{h}$ vertex-vertex, $\mathbf{i}$ vertex-random, $\mathbf{j}$ random-random

signal of intensity in the simulated images was generated simply following the dependency on $Z$ given in Eq. 3. This approximation is enough to note that, once visualized as STEM micrographs, most of the particles generated by the collisions keep a great resemblance to particles that usually are interpreted as icosahedral. Although for some of the cases this is not surprising, since the particles reached a fairly icosahedral shape, as in Fig. 9a, b, in other cases, the particles only keep some of the structural features of the original icosahedral particles (some of the tetrahedra that form a tetrahedron may be absent, for example), but at some orientations the particles will look closer to an ideal icosahedron than they really are (see, for example, Fig. 9e, i). Thus, the use of STEM simulated micrographs as a tool to elucidate the 
structural properties of real nanoparticles should not be underestimated.

\section{Conclusions}

We have investigated dynamics of the collisions between silver icosahedral nanoparticles at several impact velocities and relative orientations. The calculations were performed on relatively large nanoparticles, 2,057 atoms each. For particles of this size, impact velocities of $300 \mathrm{~m} / \mathrm{s}$ or less do not release enough energy to allow the original particles to completely coalesce, forming instead relatively stable dumbbell-like structures. The dynamics at higher velocities is quite different, and structural Ackland analysis shows that the structure of the final configuration is strongly linked to the initial orientation that the twin planes have on the parental nanoparticles. The STEM simulated micrographs of the final configurations presented in this paper may be useful for the comparison against real micrographs of similar systems, even in experiments where the thermal energy available to make the particles to coalesce comes from the interaction with external agents (heating, radiation) instead of being the direct consequence of the impact between particles. We believe that these results may contribute to understand the origin of the large variation of structural motifs on the coalescence processes of small monodisperse metallic nanoparticles.

Acknowledgments This work was supported by the Mexican Council for Science and Technology (CONACYT, Mexico), through the project CIAM 148967. Support from UANL, through the project PAICYT CE820-11 is also acknowledged.

\section{References}

Ackland GJ, Jones AP (2006) Applications of local crystal structure measures in experiment and simulation. Phys Rev B 73:054104

Antúnez-García J, Mejía-Rosales S, Pérez-Tijerina E, MontejanoCarrizales JM, José-Yacamán M (2011) Coalescence and collisions of gold nanoparticles. Materials 4(2):368-379
Baletto F, Mottet C, Ferrando R (2001) Microscopic mechanisms of the growth of metastable silver icosahedra. Phys Rev B 63:155408

Cobley CM, Skrabalak SE, Campbell DJ, Xia Y (2009) Shapecontrolled synthesis of silver nanoparticles for plasmonic and sensing applications. Plasmonics 4(2):171-179

Foiles SM, Baskes MI, Daw MS (1986) Embedded-atommethod functions for the fcc metals cu, ag, au, ni, pd, pt, and their alloys. Phys Rev B 33:7983-7991

Gracia-Pinilla M, Ferrer D, Mejía-Rosales S, Pérez-Tijerina E (2009) Size-selected ag nanoparticles with five-fold symmetry. Nanoscale res lett 4(8):896-902

Haes AJ, Van Duyne RP (2002) A nanoscale optical biosensor: sensitivity and selectivity of an approach based on the localized surface plasmon resonance spectroscopy of triangular silver nanoparticles. J Am Chem Soc 124(35): 10596-10604

Hawa T, Zachariah M (2005) Coalescence kinetics of bare and hydrogen-coated silicon nanoparticles: a molecular dynamics study. Phys Rev B 71(16):1-12

Hawa T, Zachariah M (2006) Coalescence kinetics of unequal sized nanoparticles. J Aerosol Sci 37(1):1-15

Khanal S, Bhattarai N, Velázquez-Salazar JJ, Bahena D, Soldano G, Ponce A, Mariscal MM, Mejía-Rosales S, JoséYacamán M (2013) Trimetallic nanostructures: the case of agpd-pt multiply twinned nanoparticles. Nanoscale 5(24):12456-12463

Kim JS, Kuk E, Yu KN, Kim J-H, Park SJ, Lee HJ, Kim SH, Park YK, Park YH, Hwang C-Y, Kim Y-K, Lee Y-S, Jeong DH, Cho M-H (2007) Antimicrobial effects of silver nanoparticles. Nanomed: Nanotechnol Biol Med 3(1):

95-101

Mariscal MM, Dassie SA, Leiva EPM (2005) Collision as a way of forming bimetallic nanoclusters of various structures and chemical compositions. J Chem Phys 123(18):184505

Morones JR, Elechiguerra JL, Camacho A, Holt K, Kouri JB, Ramirez JT, Yacaman MJ (2005) The bactericidal effect of silver nanoparticles. Nanotechnology 16(10):2346-2353

Perez-Tijerina E, Mejia-Rosales S, Inada H, Jose-Yacaman M (2010) Effect of temperature on AuPd nanoparticles produced by inert gas condensation. J Phys Chem C 114(15):6999-7003

Rai M, Yadav A, Gade A (2009) Silver nanoparticles as a new generation of antimicrobials. Biotechnol Adv 27(1):76-83

Sharma VK, Yngard RA, Lin Y (2009) Silver nanoparticles: green synthesis and their antimicrobial activities. Adv colloid interface sci 145(1-2):83-96

Silva LD, Machado S, Ferreira C (2011) Thin films of kanthal and aluminum for electromagnetic wave absorption. J Nano Res 14:155-163

Van McFarland AD, Duyne RP (2003) Single silver nanoparticles as real-time optical sensors with zeptomole sensitivity. Nano Lett 3(8):1057-1062 